\title{
INCIDÊNCIA E FATORES PREDISPONENTES DA MIGRAÇÃO DA FUNDOPLICATURA PELA TÉCNICA DE NISSEN-ROSSETTI NO TRATAMENTO DA DOENÇA DO REFLUXO GASTROESOFÁGICO
}

\author{
Incidence and predisponent factors for the migration of the fundoplication by Nissen-Rossetti \\ technique in the surgical treatment of GERD
}

\author{
Paulo Afonso Nunes NASSIF, Lucas Eduardo PEDRI, Priscila Reis MARTINS, Marcelo Morikuni FOAUNI, \\ Marcel da Silva JUSTEN, Michelle VARASCHIM, Denise Serpa BOPP, Osvaldo MALAFAIA
}

Trabalho realizado no Serviço de Cirurgia do Aparelho Digestivo do Hospital Universitário Evangélico de Curitiba/ Faculdade Evangélica do Paraná. Curitiba, PR, Brasil.

DESCRITORES - Fundoplicatura. Refluxo gastroesofágico
RESUMO - Racional - A doença do refluxo gastroesofágico é uma das enfermidades do trato gastrointestinal mais frequentes atualmente. A fundoplicatura de NissenRossetti é técnica operatória muito empregada para o tratamento cirúrgico, sendo a migração intratorácica da válvula uma das principais complicações. Objetivo - Avaliar a incidência da migração da fundoplicatura e seus fatores de risco. Método - Analisouse retrospectivamente 207 prontuários de pacientes submetidos à fundoplicatura videolaparoscópica pela técnica de Nissen-Rossetti para tratamento da doença do refluxo. As variáveis analisadas foram: sexo, idade, esofagite, grau de esofagite, tamanho da hérnia, alargamento da cárdia, encurtamento esofágico. Para as variáveis quantitativas, foi considerado o teste t de Student. Para as nominais, foi considerado o teste de Quiquadrado ou o teste exato de Fisher. Valores de $p<0,05$ indicaram significância estatística. Resultados - Do total, 135 eram mulheres (65,22\%) e 72 homens (34,78\%), com média de idade de 47,43 anos. O tamanho da hérnia variou entre 2 e $6 \mathrm{~cm}$. Duzentos pacientes tinham esofagite (96,62\%), sendo 113 (56,50\%) grau I, 75 grau II (37,50\%) e 12 grau III ou IV (6\%). Alargamento de cárdia e esôfago de Barrett foram vistos em 153 (73,91\%) e $13(6,28 \%)$ casos, respectivamente. Um paciente apresentava encurtamento esofágico. Dentre as mulheres, 33 (24,4\%) apresentaram migração e entre os homens, apenas seis $(8,3 \%)(p=0,005)$. A idade média dos pacientes com e sem migração foi de 54,03 e 45,89 anos, respectivamente $(p=0,001)$. Conclusão - A incidência da migração da fundoplicatura foi de $18,8 \%$. O gênero feminino e possuir maior idade influenciam na probabilidade dessa migração. O grau de esofagite, tamanho da hérnia e alargamento da cárdia não se mostraram fatores de risco para migração intratorácica da fundoplicatura.

\section{Correspondência:}

Paulo Afonso Nunes Nassif,

e-mail: paulonassif@terra.com.br

Fonte de financiamento: não há Conflito de interesses: não há

Recebido para publicação:

Aceito para publicação:

HEADINGS-Fundoplication Gastroesophageal Reflux.
ABSTRACT - Background - Gastroesophageal reflux is the gastrointestinal tract disease most frequently find nowadays. The Nissen-Rossetti fundoplication is widely used for the surgical treatment, and intrathoracic migration of the valve is the most frequent complication. Aim - To assess the incidence of the fundoplication and its risk factors. Methods - Were analyzed retrospectively medical records of 207 patients undergoing laparoscopic fundoplication by the Nissen-Rossetti technique for the treatment of reflux disease. The variables analyzed were: sex, age, esophagitis grade, size of the herniation, enlargement of the cardia and esophageal shortening. For quantitative variables, was considered the Student's t test. For the nominal, was considered the chi-square or Fisher's exact test. P values $<0.05$ were considered statistically significant. Results - Of the total, 135 were women (65.22\%) and 72 men (34.78\%) with mean age of 47.43 years. The size of the hernia varied between 2 and $6 \mathrm{~cm}$. Two hundred patients had esophagitis (96.62\%) and 113 (56.50\%) grade I, 75 grade II (37.50\%) and 12 grade III or IV (6\%). Enlargement of the cardia and Barrett's esophagus were seen in 153 (73.91\%) and $13(6.28 \%)$ cases, respectively. One patient had esophageal shortening. Among women, 33 (24.4\%) showed migration and among men, only six (8.3\%) ( $p=0.005)$. The average age of patients with and without migration was 54.03 and 45.89 years, respectively ( $p=0.001$ ). Conclusion - The incidence of the fundoplication migration was $18.8 \%$. The gender (female) and higher age influence the probability of migration. The degree of esophagitis, size of hernia and enlargement of the cardia were not risk factors for intrathoracic migration of the fundoplication 


\section{INTRODUÇÃO}

A doença do refluxo gastroesofágico (DRGE) é enfermidade crônica decorrente do fluxo retrógrado de parte do conteúdo gastroduodenal para o esôfago e/ou órgãos adjacentes, acarretando variável espectro de sintomas (esofágicos ou extra-esofágicos), associados ou não à lesões teciduais ${ }^{19}$. Ela é uma das enfermidades do trato gastrointestinal mais frequentes atualmente. Corresponde à $70 \%$ das afecções esofágicas e acomete cerca de $7-10 \%$ da população adulta. Além disso, é de grande importância médico-social pela elevada e crescente incidência e por determinar sintomas de intensidade variável, que se manifestam por tempo prolongado, podendo prejudicar consideravelmente a qualidade de vida do paciente. Também é responsável por onerar os gastos hospitalares em todo o mundo. Estima-se que a sua prevalência, em nosso meio, seja em torno de $12 \%{ }^{19}$.

O quadro de manifestações clínicas consideradas típicas da DRGE caracteriza-se, por pirose e regurgitação. Entretanto, também têm sido associadas à sintomas pulmonares e à doenças de vias aéreas inferiores como asma, tosse crônica, bronquite, pneumonia aspirativa e fibrose pulmonar idiopática. Também relaciona-se aos sinais e sintomas otorrinolaringológicos incluindo rouquidão, laringite, estenose subglótica, granuloma de prega vocal e carcinoma de laringe, além de outras manifestações extraesofágicas como dor torácica não cardíaca, erosão dentária, sinusite, faringite e apnéia do sono ${ }^{4}$.

Dentre os métodos utilizados para diagnóstico da DGRE estão: endoscopia digestiva alta, pHmetria esofágica prolongada, estudo radiológico contrastado do esôfago, manometria esofágica e impedanciometria esofágica. Cada um dos citados apresenta indicações e limitações próprias, cabendo ao médico julgá-las ${ }^{17}$.

O tratamento cirúrgico para a doença é indicado quando não há resposta satisfatória permanente dos pacientes frente à terapia medicamentosa; nas formas complicadas da doença (esôfago de Barrett, ulceração, estenose) e em pacientes com manifestações respiratórias da DRGE ${ }^{19}$.

Atualmente, o procedimento cirúrgico é realizado por meio de videolaparascopia. A primeira correção cirúrgica da DRGE por esse método foi realizada no ano de 1991 por Geagea e Dallemagne et al. Desde então, a técnica tornou-se bem aceita pelas vantagens da cirurgia minimamente invasiva, tais como menor morbidade operatória, redução do tempo de permanência hospitalar e seus custos, bem como um rápido retorno às atividades regulares ${ }^{17}$, além de melhor resultado estético ${ }^{23}$.

Nas últimas décadas, várias técnicas cirúrgicas foram desenvolvidas para tratá-la. Nos dias de hoje, a fundoplicatura de Nissen e Nissen-Rossetti são os procedimentos mais utilizados, sendo consensual sua alta eficiência ${ }^{26}$.
Muitos trabalhos de revisão associaram complicações importantes à fundoplicatura de Nissen, especialmente disfagia, incapacidade de vomitar e distensão gástrica permanente, todas relacionadas à grande competência do mecanismo valvular construído cirurgicamente. Há também o pneumotórax, que é uma das complicações intra-operatórias mais comuns, ocorrendo em 5-8\% por cento dos pacientes. Além disso, essa verdadeira vedação do estômago foi, também, associada nos casos mais graves, ao estreitamento severo esofágico, à própria perfuração do órgão, à formação de hérnias paraesofágicas, à invaginação esfagogástrica e à migração e deslizamento da válvula para o tórax. Dentre essas, a válvula intratorácica com sintomatologia persistente é a principal causa de realização de nova operação $0^{6,7,26}$.

A migração ou herniação da fundoplicatura para o tórax ocorre em $0,2-6 \%$, de acordo com a literatura descrita, podendo ocorrer precoce ou tardiamente ${ }^{6}$. A migração da válvula é mais frequente à esquerda, mas também pode ocorrer à direita ${ }^{16}$.

A sintomatologia inicial dessa migração, quando presente, se caracteriza por tosse, náusea e vômitos seguidos de quadro de desconforto abdominal, dor torácica ou abdominal e dispnéia. Os sintomas se tornam mais nítidos quando acompanhados pelo encarceramento gástrico ou volvo do estômago. O diagnóstico se baseia nas manifestações clínicas, em exames radiológicos simples de tórax e contrastados de esôfago, tomografia computadorizada de tórax e endoscopia digestiva alta, evidenciando a válvula intratorácica ${ }^{8}$.

As causas da migração não estão esclarecidas, há hipóteses de relação com a técnica utilizada aproximação inadequada do pilar direito diafragmático, mobilização insuficiente do fundo gástrico, falha na realização da sutura ou utilização de fios inadequados, ocorrência de "bucking" no momento da desintubação ou fatores de risco existentes no paciente, tais como doença prévia agravante (esofagite e periesofagite), relação com o tamanho da hérnia hiatal anterior, "esôfago curto"; de eventos decorrentes do ato cirúrgico 20,23,25.

É importante esclarecer os fatores relacionados à migração torácica da válvula de fundoplicatura por se caracterizar como um quadro de intervenção imediata podendo cursar com necrose e/ou perfuração gástrica. Nesses casos a morbidade ocorre em $70 \%$ dos pacientes e, a mortalidade, em 4,9\% deles ${ }^{8}$.

O objetivo do presente estudo foi avaliar a incidência da migração da fundoplicatura e a análise de fatores de risco para esta migração.

MÉTODO

Foram analisados em seguimento tardio de cinco anos 207 prontuários de pacientes submetidos 
à fundoplicatura videolaparoscópica com a técnica de Nissen-Rossetti para tratamento da DRGE durante o período de 2000 a 2007 no Hospital Universitário Evangélico de Curitiba/Faculdade Evangélica do Paraná, Curitiba, PR, Brasil.

Esses pacientes foram divididos em dois grupos: grupo A correspondendo aos pacientes que sofreram migração da fundoplicatura e grupo B com aqueles que não sofreram migração.

Os dados foram revisados retrospectivamente, através de uma ficha de pesquisa e de uma base de dados informatizada, a fim de analisar os possíveis fatores responsáveis para a migração da fundoplicatura.

As variáveis avaliadas foram: sexo, idade, esofagite, grau de esofagite, tamanho da hérnia, alargamento da cárdia e encurtamento esofágico.

Foram incluídos todos os pacientes com diagnóstico de DRGE - submetidos ao tratamento clínico inicial por no mínimo seis meses - com avaliação préoperatória realizada através de endoscopia digestiva alta, estudo radiológico contrastado e submetidos ao tratamento cirúrgico videolaparoscópico pela técnica de Nissen-Rossetti.

Todos receberam anti-emético de rotina no pósoperatório imediato e foram submetidos à endoscopia digestiva alta seis meses após a operação.

Considerou-se como migração da fundoplicatura o achado endoscópico que evidenciava a presença da válvula intratorácica não analisando a recorrência da doença, ou seja, a presença de sintomas da DRGE.

Para comparação das variáveis quantitativas, foi considerado o teste $t$ de Student para amostras independentes. Para as nominais, foi considerado o teste de Qui-quadrado ou o teste exato de Fisher. Valores de $p<0,05$ indicaram significância estatística. Os dados foram organizados em planilha Excel e analisados com o uso do programa computacional Statistica v.8.0.

\section{RESULTADOS}

Entre os 207 casos, foram observados que 39 pacientes tiveram migração de fundoplicatura, correspondendo a $18,8 \%$ dos casos (IC 95\%: 13,5 - 24,2).

Havia 135 mulheres $(65,22 \%)$ e 72 homens (34,78\%) com idades variando entre 21 e 79 anos (idade média de 47,43 $\pm 14,11$ anos). O tamanho da hérnia variou entre 2 e $6 \mathrm{~cm}$ (média de 3,11 $\pm 0,70 \mathrm{~cm}$ ).

Dentre as condições clínicas encontradas, $200(96,62 \%)$ pacientes tinham esofagite $(113 / 56,50 \%$ grau I, $75 / 37,50 \%$ grau II e $12 / 6 \%$ grau III ou IV). Alargamento da cárdia e esôfago de Barrett foram vistos em 153 (73,91\%) e 13 (6,28\%) casos, respectivamente. Apenas um paciente apresentava encurtamento esofágico.

No grupo de pacientes que sofreu migração da fundoplicatura (grupo A) 33 (24,4\%) eram mulheres e $6(8,3 \%)$, homens. Já dentre aqueles que não sofreram migração da fundoplicatura (grupo B) 102 (75,6\%) eram do gênero feminino e $66(91,7 \%)$ do masculino. O valor de $p$ para essa variável foi de 0,005 , possuindo significância estatística.

A idade média dos pacientes com migração foi de $54,03 \pm 14,18$ anos e dos sem migração foi de $45,89 \pm 13,69$ anos, apresentando valor estatístico significativo $(p=0,001)$.

$\mathrm{O}$ tamanho das hérnias no grupo $\mathrm{A}$ e $\mathrm{B}$ foram, respectivamente, $3,17 \pm 0,76 \mathrm{~cm}$ e 3,09 $\pm 0,69 \mathrm{~cm}(p=0,552)$.

Esofagite foi vista em todos os pacientes do grupo A e em 161 do grupo $B(p=0,352)$. O grau de esofagite também não obteve significância estatística $(p=0,193)$.

No grupo A encontraram-se 13 pacientes sem alargamento da cárdia e 26 com esse alargamento ( $p$ $=0,311$ ).

No único paciente em que foi verificada a presença de encurtamento esofágico, houve a migração da fundoplicatura.

Esôfago de Barrett foi visto em três pacientes do grupo $A$ e em 10 , do grupo $B(p=0,711)$.

\section{DISCUSSÃO}

A DRGE é condição clínica comum, que acomete cerca de $7-10 \%$ da população adulta e corresponde à aproximadamente $75 \%$ das doenças esofágicas. O tratamento inicial é habitualmente conservador, utilizando-se apenas medicamentos (sobretudo inibidores da bomba de prótons), sendo, na maioria dos casos, efetivo para controle da doença. Nos casos sem resposta satisfatória e sustentada ou naqueles com complicações (tais como esofagite severa, úlceras, estenoses ou esôfago de Barrett) há necessidade de tratamento cirúrgico ${ }^{14,16}$.

As primeiras abordagens cirúrgicas eram feitas através detécnicas abertas (laparotomia ou toracotomia). Entretanto, apenas com o advento da correção de refluxo gastroesofágico por videolaparoscopia, é que a aceitação para a terapêutica cirúrgica aumentou ${ }^{16,20}$.

Várias técnicas laparoscópicas foram idealizadas. A fundoplicatura de Nissen e sua variante idealizada por Rossetti permanecem como operações de escolha. Estudos recentes demonstraram que a fundoplicatura de Nissen-Rossetti pode ser feita laparoscopicamente em mais de $95 \%$ dos pacientes e que o controle da doença pode ser alcançado em aproximadamente $90-93 \%$ deles. Nesse estudo, os pacientes haviam sido operados pela técnica de Nissen-Rossetti2,11,14.

A grande difusão e aceitação da cirurgia laparoscópica por essa técnica pode ser confirmada pelos estudos que demonstram aumento de três a cinco vezes no número de fundoplicaturas realizadas para a DRGE na última década. Estima-se que o número de operações nos Estados Unidos aumentou de 12.000 em 1987 para 48.000 em $1998^{13}$.

Com esse aumento do número de operações laparoscópicas, falhas na operação anti-refluxo têm se tornado condição clínica comum, com suas taxas 
dobrando durante a última década. As falhas da fundoplicatura são estimadas em $6-20 \%$, sendo que os sintomas mais comuns são a recorrência do refluxo (30-60\%) e disfagia (10-20\%). Contudo, a maioria dos pacientes pode ser manejada com medicamentos, apenas precisando de reoperação em 4-6\% deles ${ }^{2,21}$.

Alguns trabalhos mostram que falha na fundoplicatura aberta ocorre em 9-30\% dos pacientes, dependendo de como a falha é definida e do tempo de acompanhamento do paciente. Na cirurgia laparoscópica essas taxas variam de $2-17 \%$, dependendo da definição de falha e a experiência do cirurgião ${ }^{13,14}$.

Estudos observaram diferença significativa no mecanismo de falha da fundoplicatura entre a operação anti-refluxo aberta e laparoscópica. Migração da fundoplicatura intacta foi a causa mais comum na laparoscopia (72,7\%). Na laparotomia, ela ocorreu mais frequentemente por ruptura da fundoplicatura. Apesar da razão para essa diferença não ser totalmente clara, tem-se sugerido que a falta de adesões na superfície anterior e posterior da fundoplicatura quando feita laparoscopicamente pode ser a causa para aumento do número das migrações ${ }^{11}$.

Há na literatura seis mecanismos de falha da cirurgia anti-refluxo laparoscópica descritos: o deslizamento da fundoplicatura, a ruptura da válvula, a herniação da fundoplicatura para o tórax, válvula muito apertada, fundoplicatura torcida e o estômago de dois compartimentos ${ }^{13}$.

A migração da fundoplicatura para o tórax tem incidência maior após a fundoplicatura laparoscópica quando comparada ao procedimento aberto. Diversas explicações possíveis têm sido levantadas: mobilização recente do paciente devido à menor dor no pósoperatório poderia induzir exercícios físicos que aumentem a pressão intra-abdominal; maior dissecção periesofágica ou menor quantidade de adesões intraperitoneais ${ }^{10,13}$.

Essa falha foi relatada pela primeira vez em 1973, por Balison et al. após abordagem transtorácica pela técnica de Nissen. Nesse estudo, $18,8 \%$ dos pacientes apresentaram válvula intratorácica como achado na endoscopia pós-operatória, não sendo levada em consideração a presença ou ausência de sintomas. $\mathrm{Na}$ literatura revisada, encontram-se taxas de migração intratorácica variando de 0,69\% até $24 \% 3,9,10,16,18,20$.

É importante ressaltar que esta condição pode ser assintomática ou sintomática, sendo que a maioria dos pacientes se encaixa no primeiro grupo. Portanto, não há relação significativa entre a recorrência de sintomas da DRGE no pós-operatório e a posição anatômica da válvula. Estudos apresentam variação entre 4,55\% a 36\% da migração como causa de sintomas e de indicação a nova operação. A válvula anti-refluxo pode apresentarse competente mesmo quando intratorácica. Por isso, nos pacientes portadores dessa circunstância, mas assintomáticos, a conduta deve ser conservadora, não

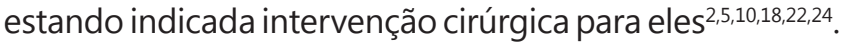

Demonstrou-se nesta série que há correlação estatística significativa entre idade $(p=0,001)$ e sexo $(p=0,005)$ com migração da fundoplicatura. Ou seja, ocorre maior número de migração nos pacientes mais idosos e naqueles do gênero feminino. Esse dado contrasta com outros estudos ${ }^{14,16,22}$.

A presença de hérnia pré-operatória ocorreu em todos os pacientes analisados, não havendo diferença significativa nos tamanhos das hérnias entre os grupos com e sem migração $(p=0,552)$. Apesar de esse resultado corroborar com outros trabalhos, alguns autores demonstram que a presença de grande hérnia hiatal préoperatória (maior que $3 \mathrm{~cm}$ ) é fator de risco significativo para a falha na operação anti-refluxo (inclusive para migração intra-torácica da válvula) 10,12,14,27.

Esôfago de Barrett foi um achado endoscópico não tão frequente $(6,28 \%)$, quando comparado às incidências relatadas na literatura que variam de $14 \%$ a $39 \% 15,19,20$. Sua relação com a migração da fundoplicatura não foi significativa $(p=0,711)$, sendo que dos 13 pacientes com ele, apenas três tiveram migração da fundoplicatura (23,1\%). Entretanto, a literatura demonstra que pacientes com esôfago de Barrett, apresentam DRGE avançada, com mais estenoses, o que contribuiria para a maior ocorrência de migração nesses pacientes ${ }^{13,14,22,25}$.

A presença de esofagite ocorreu em $96 \%$, o que corrobora os dados existentes. Em outros estudos a esofagite esteve presente em $50 \%$ a $73 \%$ dos casos. A presença e o grau de esofagite não tiveram correlação significativa com a herniação da válvula na série estudada, o que está de acordo com outros estudos publicados. Porém, existem relatos salientando que ambos poderiam atuar como risco adicional para a migração precoce da fundoplicatura $7,14,16,18,22$.

Esôfago curto é definido como a incapacidade de reduzir a junção gastroesofágica abaixo do diafragma. Vários autores sugerem que o encurtamento esofágico é fator de risco importante na ocorrência da migração da fundoplicatura devido à tensão cranial que o esôfago exerce nessa condição. No presente estudo, apenas um paciente apresentou encurtamento esofágico - não diagnosticado no pré-operatório, sendo que evoluiu com migração da fundoplicatura $(p=0,188)^{1,15,16,18}$.

Não houve também significância estatística $(p=0,311)$ na presença ou ausência de alargamento da cárdia, sendo que durante a revisão bibliográfica nenhum estudo comparava esse dado com a migração da fundoplicatura.

Algumas séries concluem que IMC elevado aumenta o risco para complicações da fundoplicatura laparoscópica de Nissen-Rossetti, visto que pacientes idosos e notadamente os obesos, têm frequentemente musculatura do hiato menos consistente e mais frágil, obrigando à dissecção mais cuidadosa e maior delicadeza na aplicação dos pontos da hiatoplastia. Porém, em outras, a obesidade não alcançou significância estatística em relação à falha da operação anti-refluxo $0^{9,18,14}$. 
Estudos recentes têm demonstrado que a atuação de forças fisiológicas estressoras no hiato do diafragma podem contribuir para a herniação da válvula. Vômitos, tosse, náuseas, esforços de vômito ou de outros tipos (levantamento de objetos pesados, esforço para urinar) são exemplos dessas forças ${ }^{13,14,15,22,25}$.

Portanto, a ocorrência de episódios de vômitos no pós-operatório precoce deve ser evitada para que a fundoplicatura não migre para o tórax. Entre as medidas para prevenção de vômitos tem-se: mudança no agente anestésico, uso de drogas antieméticas no intra-operatório, no pós-operatório imediato e no período de hospitalização, o cuidado do anestesista durante a extubação, instruções para o paciente sobre a importância das medidas anti-eméticas, orientações dietéticas e conscientização da não realização de esforço físico excessivo. Uma dose simples de corticóide EV também tem demonstrado redução significativa na incidência e severidade das náuseas e vômitos no pósoperatório ${ }^{14,18}$.

Ressalta-se, também, o fato de que a adoção de anti-eméticos em todos os pacientes no pósoperatório explicaria a ausência de migração precoce da fundoplicatura. O serviço em que foi realizado esse procedimento também utiliza esses medicamentos rotineiramente, entretanto há migração precoce da fundoplicatura em alguns casos, não sendo uma explicação aplicável neste trabalho ${ }^{25}$.

O sucesso da operação anti-refluxo está diretamente relacionado à indicação da mesma, escolha do tipo de procedimento, meticulosa técnica cirúrgica, experiência do cirurgião e seleção criteriosa do paciente. Técnica cirúrgica inadequada, escolha incorreta do procedimento e pacientes selecionados não corretamente foram causas de falha de $42 \%$ a $66 \%$ dos casos relatados na literatura ${ }^{2,7,9,14,20,25}$.

Em relação à qualidade técnica da operação antirefluxo, três fatores principais parecem estar envolvidos para maior ocorrência da migração da fundoplicatura: fechamento inadequado dos pilares diafragmáticos; a não-fixação da válvula na superfície inferior no diafragma e em outros tecidos periesofágicos; mobilização insuficiente do fundo gástrico e utilização de fios inadequados. Por isso, alguns trabalhos sugerem o fechamento posterior dos pilares diafragmáticos com fios não-absorvíveis e fixação da válvula no esôfago e superfície inferior do diafragma $7,15,16,20,22,28$.

\section{CONCLUSÃO}

A incidência da migração da fundoplicatura foi de $18,8 \%$. O gênero feminino e possuir maior idade influenciam na probabilidade dessa migração. $\mathrm{O}$ grau de esofagite, tamanho da hérnia e alargamento da cárdia não se mostraram fatores de risco para migração intratorácica da fundoplicatura.
REFERÊNCIAS

1. Bochkarev V, Lee YK, Vitamvas M, Oleynikov D. Short esophagus: how much length can we get? Surg Endosc. 2008, 22 (10): 21232127

2. Curet MJ, Josloff RK, Schoeb O, Zucker KA. Laparoscopic reoperation for failed antireflux procedures. Arch Surg. 1999, 134 (5): 559-563

3. Floch NR, Hinder RA, Klingler PJ, Branton SA, Seelig MH, Bammer T, Filipi CJ. Is laparoscopic reoperation for failed antireflux surgery feasible? Arch Surg. 1999, 134 (7): 733-737

4. Funes $H L X$, Anai GK, Santos MCL, Leite APM, Salvador FC. Videolaparoscopia no tratamento da doença por refluxo gastroesofágico. Rev Col Bras Cir. 2000; 27 (5): 312-315.

5. Furnée EJB, Draaisma WA, Broeders IAMJ, Smout AJPM, Gooszen HG. Surgical reintervention after antireflux surgery for gastroesophageal reflux disease. Arch Surg. 2008; 143 (3): 267274

6. Godoy AQ, Morioka RH, Fonseca PC, Godoy ARS, Godoy GRS. Migração precoce da fundoplicatura após tratamento cirúrgico videolaparoscópico da doença do refluxo gastroesofágico. Rev Col Bras Cir. 2003, 30 (3): 194-197

7 Granderath FA, Kamolz T, Schweiger UM, Pointner R. Failed antireflux surgery: quality of life and surgical outcome after laparoscopic refundoplication. Int J Colorectal Dis. 2003; 18 (3): 248-253.

8. Gurski RR, Rosa ARP, Valle E, Borba MA, Valiati AA. Manifestações extra-esofágicas da doença do refluxo gastroesofágico. J Bras Pneumol. 2006; 32 (2): 150-160.

9. Hahnloser D, Schumacher M, Cavin R, Cosendey B, Petropoulos P. Risk factors for complications of laparoscopic Nissen fundoplication. Surg Endosc. 2002, 16 (1): 43-47

10. Hainaux B, Sattari A, Coppens $E$, Sadeghi N, Cadière GB. Intrathoracic migration of the wrap after laparoscopic Nissen fundoplication: radiologic evaluation. AJR Am J Roentgenol. 2002, 178 (4): 859-862

11. Heniford BT, Matthews BD, Kercher KW, Pollinger $H$, Sing RF. Surgical experience in fifty-five consecutive reoperative fundoplications. Am Surg. 2002, 68 (11): 949-954

12. Horgan S, Pohl D, Bogetti D, Eubanks T, Pellegrini C. Failed antireflux surgery: what have we learned from reoperations? Arch Surg. 1999, 134 (8): 809-815

13. Hunter JG, Smith CD, Branum GD, Waring JP, Trus TL, Cornwell M, Galloway K. Laparoscopic fundoplication failures: patterns of failure and response to fundoplication revision. Ann Surg. 1999, 230 (4): 595-604

14. Igbal A, Awad Z, Simkins J, Shah R, Haider M, Salinas V, Turaga K, Karu A, Mittal SH, Fillipi CJ. Repair of 104 failed anti-reflux operations. Ann Surg. 2006, 244 (1): 42-51

15. Khajanchee YS, O'Rourke R, Cassera MA, Gatta P, Hansen PD, Swanström LL. Laparoscopic reintervention for failed antireflux surgery: subjective and objective outcomes in 176 consecutive patients. Arch Surg. 2007, 142 (8): 785-792

16. Liboni NS, Silva RA. Migração de fundoplicatura e do estômago para o hemitórax direito como complicação imediata de cardioplastia videolaparoscópica em doente portador de amiloidose. Rev Col Bras Cir. 2001, 28 (6): 456-458

17. Lopes LR, Brandalise NA, Andreollo NA, Leonardi LS. Tratamento cirúrgico videolaparoscópico da doença do refluxo gastroesofagiano: técnica de Nissen modificada - resultados clínicos e funcionais. Rev Assoc Med Bras. 2001; 47 (2): 141-148.

18. Malafaia O, Nassif PAN, Ribas Filho JM, Czeczko NG, Campos $A C L$, Naufel Junior CR. Migração da fundoplicatura: profilaxia. In: Coelho JC, organizador. Atualização em cirurgia do aparelho digestivo e coloproctologia. São Paulo: Frontis; 2004. v.1, p.5-9

19. Nasi A, Moraes-Filho JPP, Cecconello I. Doença do refluxo gastroesofágico: revisão ampliada. Arq Gastroenterol. 2006; 43 (4): 334-341. 
20. Neuhauser B, Hinder RA. Laparoscopic reoperation after failed antireflux surgery. Surg Innov. 2001, 8 (4): 281-286

21. Ohnmacht GA, Deschamps C, Cassivi SD, Nichols III FC, Allen MS, Schleck CD, Pairolero PC. Failed antireflux surgery: results after reoperation. Ann Thorac Surg. 2006, 81 (6): 2050-2054

22. Patti MG, Arcerito M, Feo CV, Pinto M, Tong J, Gantert W, Tyrrell D, Way LW. An analysis of operations for gastroesophageal reflux disease. Arch Surg. 1998; 133 (6): 600-607.

23. Pinto KF, Ferrante SMR, Siviero I, Meio IB, Turcatel MA, Forny D. Estudo comparativo entre as técnicas de nissen e Boix-Ochoa em ratos. Rev Col Bras Cir. 2007; 34 (6): 407-411.

24.Soper NJ, Dunnegan D. Anatomic fundoplication failure after laparoscopic antireflux surgery. Ann Surg. 1999; 229 (5): 669.
25. Spechler, SJ. The management of patients who have "failed" antireflux surgery. Am J Gastroenterol. 2004;99(3):552-561.

26. Townsend MC, Beauchamp RD, Evers BM, Mattox KL. Sabiston, tratado de cirurgia: a base biológica da moderna prática cirúrgica. $17^{a}$. ed. Rio de Janeiro: Elsevier; 2005. Vol. 1, p. 1158-1160

27. Voitk A, Joffe J, Alvarez C, Rosenthal G. Factors contributing to laparoscopic failure during the learning curve for laparoscopic Nissen fundoplication in a community hospital. J Laparoendosc Adv \}Surg Tech A. 1999, 9 (3): 243-248

28. Wu JS, Dunnegan, DL, Luttmann DR, Soper NJ. The influence of surgical technique on clinical outcome of laparoscopic Nissen fundoplication. Surg Endosc. 1996, 10 (12): 1164-1170 